\title{
Satirical contribution: COVID-19 research updates: does wearing tinfoil hats pose neurodegenerative threats to conspiracists and the general public?
}

\author{
I. M. Portant ${ }^{1} \cdot$ R. E. Sults ${ }^{1}$ \\ ๑) Springer-Verlag GmbH Germany, part of Springer Nature 2021
}

\section{Introduction}

After our paper on non-linear endocrine no-dose effects had triggered an unprecedented seismic paradigm shift in the otherwise tranquil world of toxicology (Portant and Sults 2019), the authors' hard time with funding acquisition was fortunately ended by the corona pandemic, since all research projects, no matter how moronic, were suddenly drowned in money if they only had "COVID-19" in their name. Equipped with rich funding, we initially thought of developing an expensive, completely useless corona warning app but had to realize that we had already been outdone. Then we thought of a children's straitjacket with an integrated artificial intelligence-controlled EEG, which gives the children, malicious drivers of the deadly pandemic, a $1000 \mathrm{~V}$ jolt whenever they even think about taking off their everyday and night mask. Here too we had to realize that the world had already left us behind by horse lengths with brilliant, far more sadistic solutions. We were deeply impressed by politicians who almost daily introduced new contradictory regulations to fight the pandemic. The wisdom of those measures was enthusiastically supported by an applauding public, whereas a minority of tinfoil hat (synonym: aluminum foil hat) conspiracists insisted on an evidence-free standpoint of non-existence of the virus. At this point, in our desperate search for a research niche related to toxicology and alternative methods, we finally recognized our calling.

Aluminum exposure is believed to have the potential to adversely affect human health, including the induction of

This is a related article to https://doi.org/10.1007/s00204-01902481-y.

\footnotetext{
I. M. Portant

graf@ifado.de

1 Awkward Medical School/Moron Institute of Technology, Dortmund, Germany
}

neurodegenerative diseases associated with cognitive dysfunction. Previous studies have identified aluminum-containing food contact materials, especially in conjunction with acidic foodstuff and high-temperature food preparation, as one of the major sources of aluminum exposure. Other routes of exposure, e.g. dermal uptake of aluminum from cosmetics, may also play a relevant role.

So-called tinfoil hats, usually made from aluminum foil, are headgear which is worn by conspiracists to protect them from governmental brainwashing, chemtrails, abduction by extraterrestrials, and other paranormal threats. Wise politicians from major political parties have discovered that tinfoil hats are worn by the vast majority of protesters present at demonstrations against governmental anti-COVID-19 measures in Germany. This fact raises severe concerns about increased aluminum exposure due to dermal and transcerebral uptake from tinfoil hats, and possibly related health issues for the conspiracists. These people may be at particular risk for neuronal aluminum effects for two reasons: first, the tinfoil hat as possible source of aluminum is worn for longer periods, in close vicinity to the cerebrum. Second, as notorious hotheads the conspiracists might feature elevated skull temperature during the demonstrations, thus facilitating the migration of aluminum. Therefore, a joint research project of the Awkward Medical School and the Moron Institute of Technology has investigated potential health risks, especially impairment of brain functions, associated with the wearing of tinfoil hats at protests against anti-COVID-19 measures, and we will here for the first time report groundbreaking results with far-reaching implications. 


\section{Materials and methods}

\section{In vitro cell culture}

Cultures of iPSC (imPotentStemCell)-derived neuronal cells were grown in standard medium, and incubated with miniaturized tinfoil hats (Fig. 1a). To mimic the conditions at a demonstration against anti-COVID-19 measures, incubation time was set to $3 \mathrm{~h}$ and additional experiments were run at elevated temperature. Subsequently, cell viability was assessed using previously described assays. We are tired of explaining them in detail again.

\section{Self-experiment}

In a heroic self-experiment, the authors exposed themselves to four common-sense inducers of intellectual dysfunction: smartphones, trash TV, a homeopathic low-dose cocktail of chemicals, and tinfoil hats. To observe dose dependency, exposure was performed for $3 \mathrm{~h}$ to one or two smartphones, one or two talk shows with commercials, a homeopathic low-dose chemical cocktail of dangerous chemicals or a small, medium, or large tinfoil hat. The authors reported effects by answering the question if they felt more stupid after exposure.

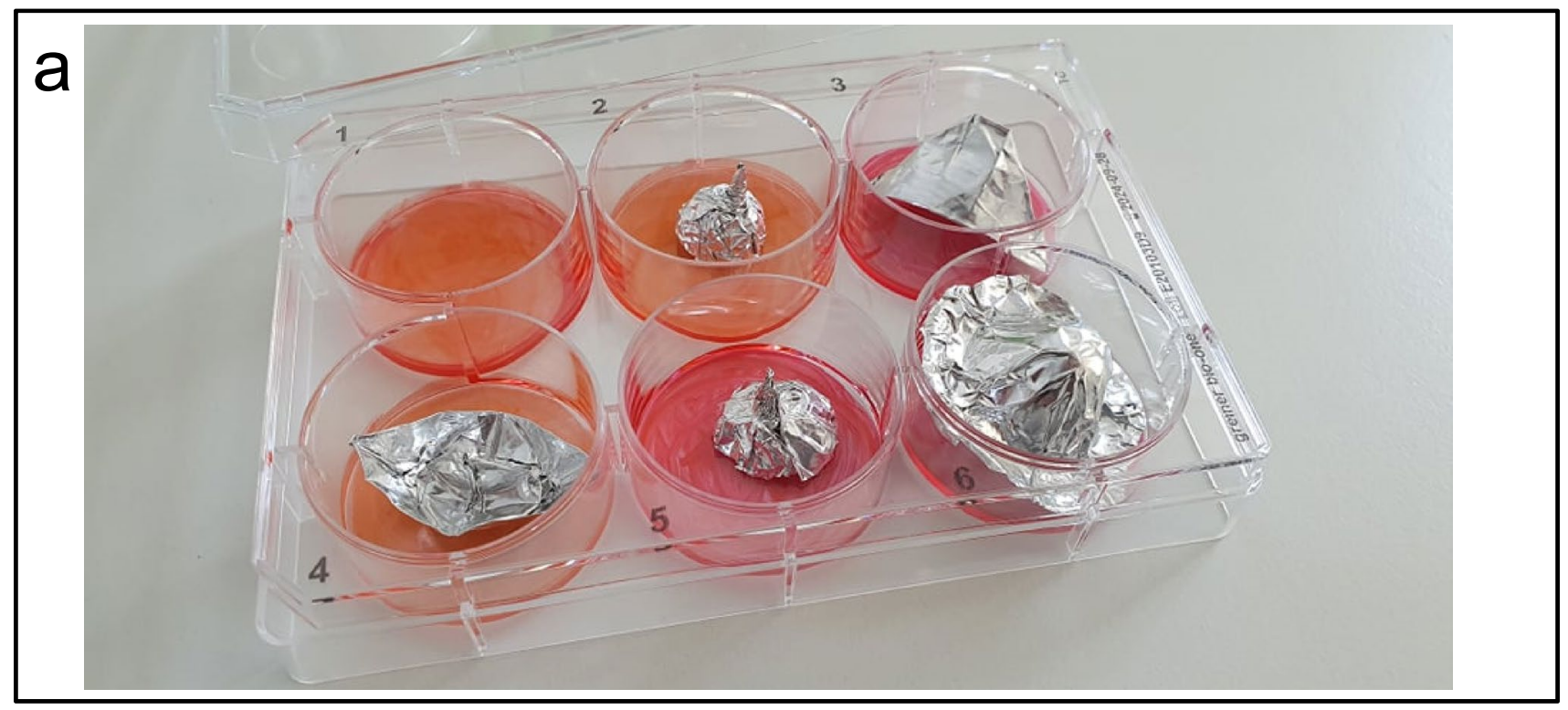

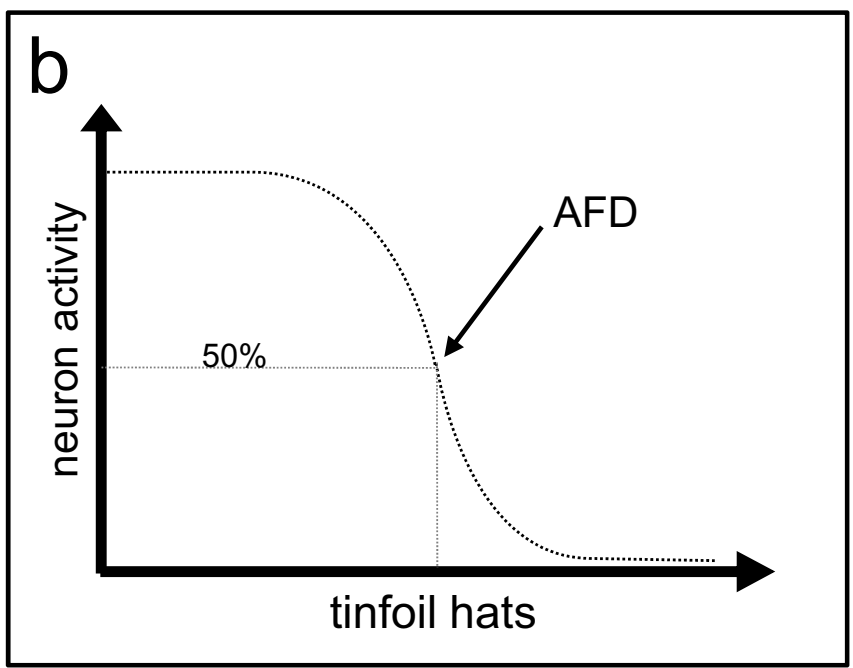

Fig. 1 In vitro analysis of tinfoil hat toxicity to human neuronal cells. a Experimental cell culture system setup with miniaturized tinfoil hats. b Correlation of in vitro tinfoil hat exposure and neuronal activity. The acute flapdoodle dose (AFD) indicates the dose at which $50 \%$

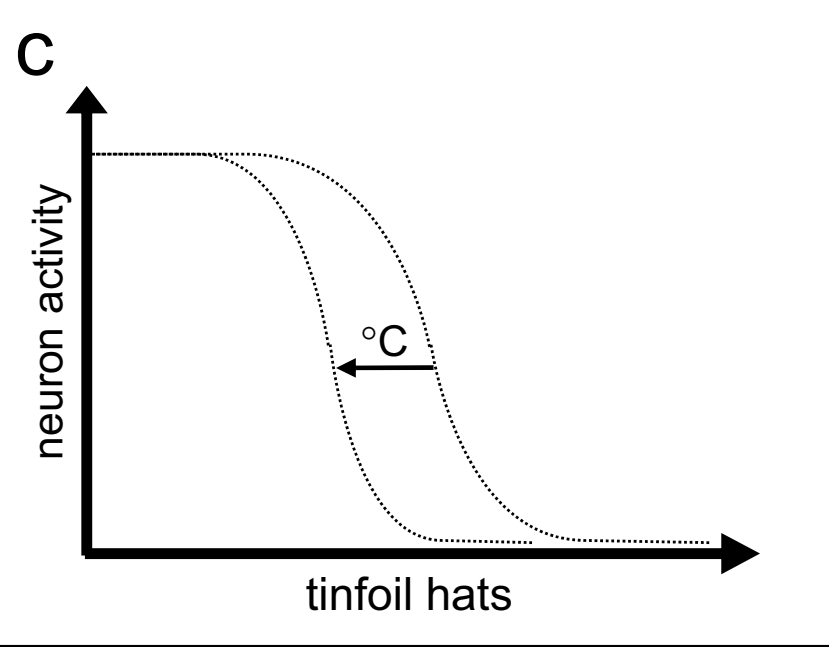

of neuronal activity is lost. c Toxicity is exacerbated by elevated temperature, mimicking the situation at anti-COVID-19 measures protests 


\section{In vivo study with conspiracists}

Neuronal function in terms of cognitive capacities of volunteer conspiracists was measured in an FOB (freaky observational battery) that included a parliament escalade and a fake news-generating messenger tool for performing simple cognitive tasks. Conspiracists were divided into two groups, one wearing and one not wearing tinfoil hats. Results were correlated with the duration of tinfoil hat exposure and the hat size, using the patented and consequently unpublishable Portant and Sults multi-parametric linear non-monotonic regression model.

\section{Results and discussion}

\section{Miniaturized tinfoil hats impair neuronal cell viability in vitro}

Initially, an in vitro experiment was carried out to investigate the effects of tinfoil hats on human neuronal cells. As isolation of primary neurons from conspiracists was not successful (data not shown), iPSC-derived neurons were used for the study. As evident from Fig. 1b, a dose-dependent loss of neuronal cell viability caused by exposure to tinfoil hats was recorded. Modeling of the dose-response curve allowed us to derive the AFD (acute flapdoodle dose) corresponding to a $50 \%$ loss of neuron function. Increased temperature potentiated the adverse effects of the tinfoil hats (Fig. 1c). Taken together, the in vitro results strongly suggest that wearing tinfoil hats may pose a substantial risk to mental health and intellectual properties.

\section{Self-experiment demonstrates in vitro intellectual dysfunction caused by tinfoil hats}

To verify this hypothesis in vivo, the authors performed a self-experiment to test the correlation of brain function and tinfoil hat exposure. As shown in Fig. 2a, there was a clear and dose-dependent decrease in intellectual capacities of scientists by wearing tinfoil hats. All the other tested factors, however, did not decrease the extraordinary intellectual capacities of the authors (data not shown).

\section{Unexpected lack of intellectual dysfunction by tinfoil hat exposure in conspiracists}

Volunteer conspiracists were then recruited for an in vivo test series. Aluminum body burden of the probands was analyzed in quiescent state (without tinfoil hat), after wearing a tinfoil hat for $3 \mathrm{~h}$, and after wearing a tinfoil hat for $3 \mathrm{~h}$ at a demonstration. Brain activities were assessed by FOB as described in the methods section. In line with our hypothesis, Fig. 2b illustrates the substantial increase in aluminum body burden of the test persons by the tinfoil hats, with the highest values obtained in the tinfoil hat plus demonstration scenario, thereby confirming our hypothesis and in vitro findings. Surprisingly, and in sharp contrast to the findings obtained in vitro, no decrease in relative brain function was observed in the tested population (Fig. 2c). However, a different picture emerged when the focus was not set to relative alterations of brain function, but to the analysis of the absolute intellectual capacity of the test persons: Fig. $2 \mathrm{~d}$ demonstrates that the absolute neuronal activities of the conspiracists participating in our study were all far below the lower level of intellectual subsistence. A further decrease caused by tinfoil hats was thus just not possible, thereby elucidating the observed lack of adverse effects of tinfoil hats in our study. Moreover, this finding may provide an explanation why an isolation of viable conspiracist-derived neurons for in vitro cultivation was not successful (see above).

\section{Novel tools for in vivo-in vitro extrapolation}

To bridge the gap between in vivo and in vitro results, we first checked our in vitro results by applying the $3 \mathrm{R}$ principle (rephrase, retry, resign). However, the discrepancy between in vitro and in vivo data could not be cleared up (data not shown). Therefore, we suggest the introduction of a new parameter for in vitro-in vivo trans-species extrapolation in neurotoxicology, the so-called ANON (Absolute NONsense) quotient $\left(\mathrm{Q}_{\mathrm{ANON}}\right)$. Roughly, $\mathrm{Q}_{\mathrm{ANON}}$ is inversely correlated with the intelligence quotient of the test person or species in vivo. We have derived default values for $\mathrm{Q}_{\mathrm{ANON}}$, which can be applied for better interpretation of in vitro neuronal activity data derived from experiments with normal human cells (Table 1). The values from in vitro just need to be divided by the $\mathrm{Q}_{\mathrm{ANON}}$ to obtain the corresponding brain activity of the relevant species in vivo.

Taken together, the presented data show that conspiracists are not at risk of neuronal impairment when wearing tinfoil hats. Further studies, however, are required to assess the toxicity of tinfoil hat wearers to average citizens at the whole population level. 

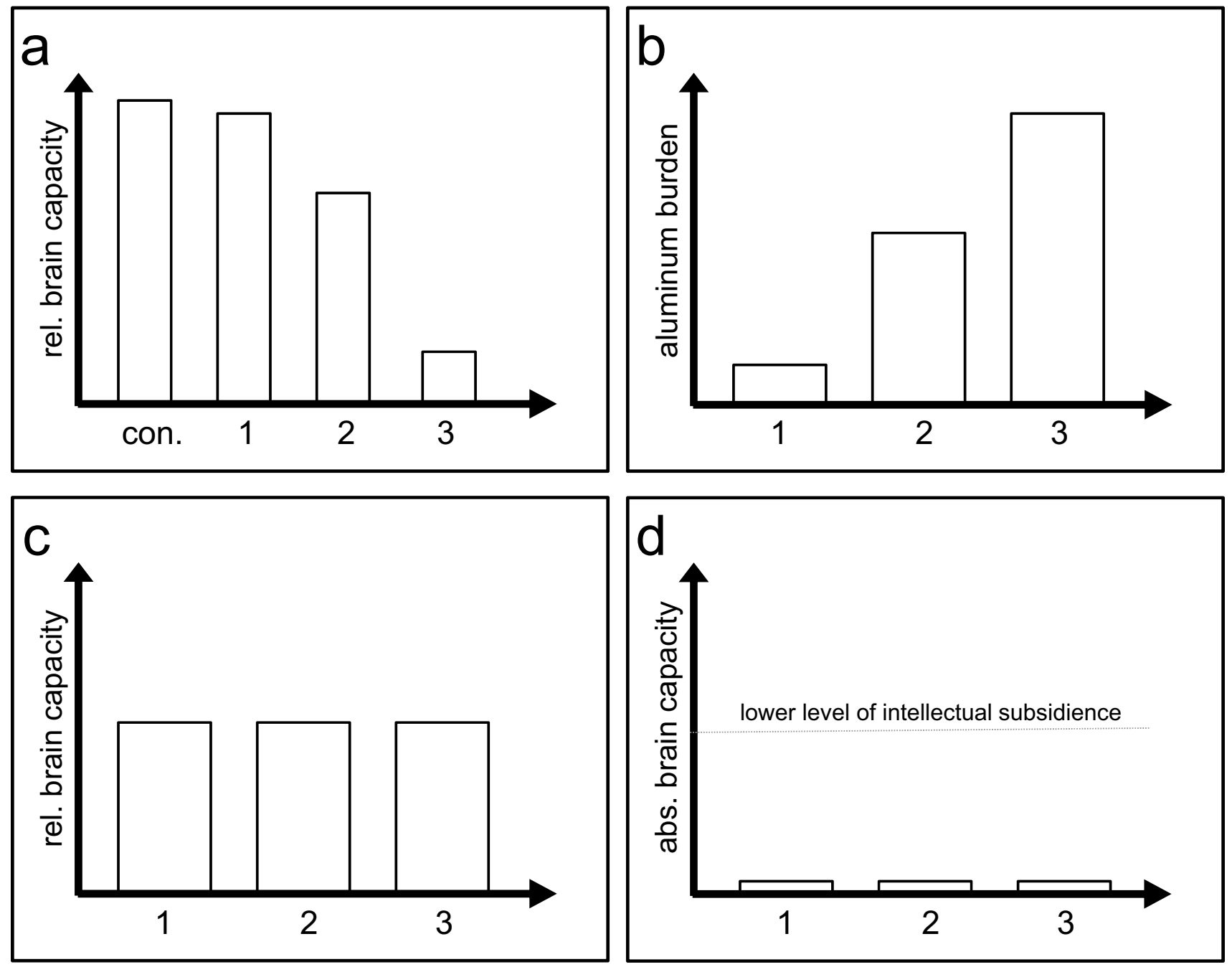

Fig. 2 In vivo study of neuronal impairment in scientists and volunteer conspiracists. a Tinfoil hat exposure leads to temporary intellectual dysfunction in scientists, as determined in a self-experiment with differently sized tinfoil hats (con., control without hat; 1-3, small, medium and large tinfoil hat). b-d Results yielded with conspiracists. Probands were analyzed either in normal quiescent state without tinfoil hat (1), after $3 \mathrm{~h}$ of wearing a tinfoil hat (2), or after $3 \mathrm{~h}$ of wear-

Table $1 \mathrm{Q}_{\mathrm{ANON}}$ values for different species

\begin{tabular}{lc}
\hline Species or test person in vivo & ANON quotient \\
\hline Average human & 1 \\
Chimpanzee & 3 \\
Zebrafish & 50 \\
Conspiracist or politician & 1000 \\
\hline
\end{tabular}

Acknowledgements We acknowledge that this work should probably have been discussed with an ethics board. Unfortunately, the authors did not receive any funding from Bill Gates, even though we repeatedly sent him our account number.

ing a tinfoil hat at a demonstration of conspiracists (3). Mean values are shown. b Aluminum body burden is increased by wearing tinfoil hats. c Irrespective of aluminum exposure, the relative brain capacity of the test persons remains constant. d Plotting of absolute brain activity demonstrates that neuronal activity of the test persons was below the lower level of intellectual subsidience and could thus not be further decreased by tinfoil hat exposure

\section{Declarations}

Conflict of interest The authors declare that conflict is of interest.

\section{Reference}

Portant IM, Sults RE (2019) Non-linear endocrine no-dose effects-towards a new paradigm in toxicology. Arch Toxicol 93:1773-1774

Publisher's Note Springer Nature remains neutral with regard to jurisdictional claims in published maps and institutional affiliations. 